\title{
Maximal acceleration constant puzzle for DIRAC experiment in CERN
}

\author{
Miroslav Pardy \\ Department of Physical Electronics \\ Masaryk University \\ Kotlářská 2, 61137 Brno, Czech Republic \\ e-mail:pamir@physics.muni.cz
}

January 22, 2019

\begin{abstract}
We determine the nonlinear group of transformations between coordinate systems which are mutually in a constant symmetrical uniform acceleration. The maximal acceleration limit is a constant which follows from the logical necessity and the kinematical necessity of the system motion and it is an analogue of the maximal velocity in special relativity. The Pardy acceleration constant is not the same as the Caianiello acceleration constant in quantum mechanics and Lambiase acceleration constant in the Riemann space-time and this situation forms the serious puzzle in physics after the theta-tau puzzle in particle physics and Hawking black hole puzzle in cosmology. The author transformations of the accelerated systems is related to the Orlov transformations. The DIRAC experiment in CERN with pionium in the strong electrical field is discussed.
\end{abstract}

\section{Introduction}

The maximal acceleration constant by Pardy and Caianiello can be considered as the integral part of the theory of physical constants in classical physics and particle physics. The term of fundamental physical constant is reserved for physical quantities which, according to the current state of knowledge, are regarded as immutable and as non-derivable from more fundamental principles. Notable examples are the speed of light c, and the gravitational constant G. The maximal acceleration constant can be determined by appropriate experiments. One of such experiment is the Dirac experiment in CERN with pionoum.

The pionium atom $A_{2 \pi}$ is a hydrogen-like bound state of a $\pi^{+}$and a $\pi^{-}$meson. This atom decays predominantely strongly into two uncharged $\pi^{0} \pi^{0}$. The transition matrix of this decay is directly proportional to the difference of the two S-wave $\pi \pi$-scattering lengths with isospin 0 and 2: $a_{0}-a_{2}$. The pionium lifetime $\tau$ is inversely proportional to the squared scattering length difference. In the framework of chiral perturbation theory (ChPT), the 
scattering length difference has been precisely calculated. The DIRAC experiment enables to determine the lifetime of pionium and hence the difference in the two scattering lengths to provide the possibility to check the ChPT predictions in a model independent way (Schuetz, 2003). However, after inserting the pionium atoms into the strong electrical field we get the pion accelerated systems and we are prepared to measure the maximal constant acceleration defined by Pardy and by Caianiello, or, by Lambiase.

\section{Lorentz transformation}

To be pedagogically clear let us remind, at first, some ingredients of the special theory of relativity velocity and acceleration. The Lorentz transformation between two inertial coordinate systems $S(0, x, y, z)$ and $S^{\prime}\left(0^{\prime}, x^{\prime}, y^{\prime}, z^{\prime}\right)$ (where system $S^{\prime}$ moves in such a way that $x$-axes converge, while $y$ and $z$-axes run parallel and at time $t=t^{\prime}=0$ for the origin of the systems $O$ and $O^{\prime}$ it is $O \equiv O^{\prime}$ ) is as follows:

$$
x^{\prime}=\gamma(v)(x-v t), \quad y^{\prime}=y, \quad z^{\prime}=z^{\prime}, \quad t^{\prime}=\gamma(v)\left(t-\frac{v}{c^{2}} x\right),
$$

where

$$
\gamma(v)=\left(1-\frac{v^{2}}{c^{2}}\right)^{-1 / 2} .
$$

The infinitesimal form of this transformation is evidently given by differentiation of the every equation. Or,

$$
d x^{\prime}=\gamma(v)(d x-v d t), \quad d y^{\prime}=d y, \quad d z^{\prime}=d z, \quad d t^{\prime}=\gamma(v)\left(d t-\frac{v}{c^{2}} d x\right) .
$$

It follows from eqs. (3) that if $v_{1}$ is velocity of the inertial system 1 with regard to $S$ and $v_{2}$ is the velocity of the inertial systems 2 with regard to 1 , then the relativistic sum of the two velocities is

$$
v_{1} \oplus v_{2}=\frac{v_{1}+v_{2}}{1+\frac{v_{1} v_{2}}{c^{2}}} .
$$

The infinitesimal form of Lorentz transformation (3) evidently gives the Lorentz length contraction and time dilation. Namely, if we put $d t=0$ in the first equation of system (3), then the Lorentz length contraction follows in the infinitesimal form $d x^{\prime}=\gamma(v) d x$. Or, in other words, if in the system $S^{\prime}$ the infinitesimal length is $d x^{\prime}$, then the relative length with regard to the system $S$ is $\gamma^{-1} d x^{\prime}$. Similarly, from the last equation of (3) it follows the time dilatation for $d x=0$. Historical view on this effect is in the Selleri article (Selleri, 1997).

\section{Uniformly accelerated frames with space-time symmetry}

Let us take two systems $S(0, x, y, z)$ and $S^{\prime}\left(0^{\prime}, x^{\prime}, y^{\prime}, z^{\prime}\right)$, where system $S^{\prime}$ moves in such a way that $x$-axes converge, while $y$ and $z$-axes run parallel and at time $t=t^{\prime}=0$ for the beginning of the systems $O$ and $O^{\prime}$ it is $O \equiv O^{\prime}$. Let us suppose that system $S^{\prime}$ moves relative to some basic system $B$ with acceleration $a / 2$ and system $S^{\prime}$ moves relative to system $B$ with acceleration $-a / 2$. It means that both systems moves one another with acceleration $a$ and are equivalent because in every system it is possibly to observe the force caused by the acceleration $a / 2$. In other words no system is inertial. The physical realization of such two accelerated frames is the frames connected with the charge $\mathrm{Q}$ and $-\mathrm{Q}$ acceleratd by the homogenous electric field (Orlov, 2014a; 2014b; 2014c). 
Now, let us consider the formal transformation equations between two systems. At this moment we give to this transform only formal meaning because at this time, the physical meaning of such transformation is not known. On the other hand, the consequences of the transformation will be shown very interesting. The first published derivation of such transformation by the standard way was given by author (Pardy, 2003; 2004; 2005), and the same transformations were submitted some decades ago (Pardy, 1969; 1974). The old results can be obtained if we perform transformation

$$
t \rightarrow t^{2}, \quad t^{\prime} \rightarrow t^{\prime 2}, \quad v \rightarrow \frac{1}{2} a, \quad c \rightarrow \frac{1}{2} \alpha
$$

in the original Lorentz transformation (1). We get:

$$
x^{\prime}=\Gamma(a)\left(x-\frac{1}{2} a t^{2}\right), \quad y^{\prime}=y, \quad z^{\prime}=z, \quad t^{\prime 2}=\Gamma(a)\left(t^{2}-\frac{2 a}{\alpha^{2}} x\right)
$$

with

$$
\Gamma(a)=\frac{1}{\sqrt{1-\frac{a^{2}}{\alpha^{2}}}} .
$$

We used practically new denotation of variables in order to get the transformation (6) between accelerated systems.

The transformations (6) form the one-parametric group with the parameter $a$. The proof of this mathematical statement can be easy performed if we perform the transformation $T_{1}$ from $S$ to $S^{\prime}$, transformation $T_{2}$ from $S^{\prime}$ to $S^{\prime \prime}$ and transformation $T_{3}$ from $S$ to $S^{\prime \prime}$. Or,

$$
\begin{gathered}
x^{\prime}=x^{\prime}\left(x, t ; a_{1}\right), \quad t^{\prime}=t^{\prime}\left(x, t ; a_{1}\right), \\
x^{\prime \prime}=x^{\prime \prime}\left(x^{\prime}, t^{\prime} ; a_{2}\right), \quad t^{\prime \prime}=t^{\prime \prime}\left(x^{\prime}, t^{\prime} ; a_{2}\right) .
\end{gathered}
$$

After insertion of transformations (8) into (9), we get

$$
x^{\prime \prime}=x^{\prime \prime}\left(x, t ; a_{3}\right), \quad t^{\prime \prime}=t^{\prime \prime}\left(x, t ; a_{3}\right),
$$

where parameter $a_{3}$ is equal to

$$
a_{3}=\frac{a_{1}+a_{2}}{1+\frac{a_{1} a_{2}}{\alpha^{2}}} .
$$

The inverse parameter is $-a$ and parameter for identity is $a=0$. It may be easy to verify that the final relation for the definition of the continuous group transformation is valid for our transformation. Namely (Eisenhart, 1943):

$$
\left(T_{3} T_{2}\right) T_{1}=T_{3}\left(T_{2} T_{1}\right)
$$

The physical interpretation of this nonlinear transformations is the same as in the case of the Lorentz transformation only the physical interpretation of the invariant function $x=(1 / 2) \alpha t^{2}$ is different. Namely it can be expressed by the statement: if there is a physical signal in the system $S$ with the law $x=(1 / 2) \alpha t^{2}$, then in the system $S^{\prime}$ the law of the process is $x^{\prime}=(1 / 2) \alpha t^{\prime 2}$, where $\alpha$ is the constant of maximal acceleration. It is new constant and cannot be constructed from the known physical constants.

Let us remark, that it follows from history of physics, that Lorentz transformation was taken first as physically meaningless mathematical object by Larmor, Voigt and Lorentz and later only Einstein decided to put the physical meaning to this transformation and to the invariant function $x=c t$. We hope that the derived transformation will appear as physically meaningful. 
Using relations $t \leftarrow t^{2}, \quad t^{\prime} \leftarrow t^{\prime 2}, \quad v \leftarrow \frac{1}{2} a, \quad c \leftarrow \frac{1}{2} \alpha$, the nonlinear transformation is expressed as the Lorentz transformation forming the one-parametric group. This proof is equivalent to the proof by the above direct calculation. The integral part of the group properties is the so called addition theorem for acceleration.

$$
a_{3}=a_{1} \oplus a_{2}=\frac{a_{1}+a_{2}}{1+\frac{a_{1} a_{2}}{\alpha^{2}}} .
$$

where $a_{1}$ is the acceleration of the $S^{\prime}$ with regard to the system $S, a_{2}$ is the acceleration of the system $S^{\prime \prime}$ with regard to the system $S^{\prime}$ and $a_{3}$ is the acceleration of the system $S^{\prime \prime}$ with regard to the system $S$. The relation (13), expresses the law of acceleration addition theorem on the understanding that the events are marked according to the relation (6).

If $a_{1}=a_{2}=a_{3}=\ldots .+a_{i}=a$, for $i$-th accelerated carts which rolls in such a way that the first cart rolls on the basic cart, the second rolls on the first cart and so on, then we get for the sum of $i$ accelerated carts the following formula

$$
a_{\text {sum }}=\frac{1-\left(\frac{1-a / \alpha}{1+a / \alpha}\right)^{i}}{1+\left(\frac{1-a / \alpha}{1+a / \alpha}\right)^{i}},
$$

which is an analogue of the formula for the inertial systems (Lightman et al., 1975).

\section{Discussion}

In formula (14) as well as in the transformation equation (6) appears constant $\alpha$ which cannot be calculated from the theoretical considerations, or, constructed from the known physical constants (in analogy with the velocity of light). What is its magnitude can be established only by experiments. The notion maximal acceleration was introduced some decades ago by author (Pardy, 1974). Caianiello (1981) introduced it as some consequence as some consequence of of quantum mechanics and Landau theory of fluctuations. Revisiting view on the maximal acceleration was given by Papini (2003). At present time it was argued by Lambiase et al. (1999) that maximal acceleration determines the upper limit of the Higgs boson and that it gives also the relation which links the mass of $W$-boson with the mass of the Higgs boson. The LHC and HERA experiments presented different answer to this problem.

The maximal acceleration constant which was derived here is kinematic one and it differs from the Caianiello (1981) definition following from quantum mechanics. Our constant cannot be determined by the system of other physical constants. It is an analogue of the numeric velocity of light which cannot be composed from others physical constants, or, the Heisenberg fundamental length in particle physics. The nonlinear transformations (13) changes the Minkowski metric

$$
d s^{2}=c^{2} d t^{2}-d x^{2}-d y^{2}-d z^{2}
$$

to the new metric with the Riemann form. Namely:

$$
d s^{2}=\alpha^{2} t^{2} d t^{2}-d x^{2}-d y^{2}-d z^{2}
$$

and it can be investigated by the methods of differential geometry. So, equations (13) can form the preamble to investigation of accelerated systems. It was shown by Orlov that the nonlinear group of transformations by author are related to the Orlov transformations for accelerated frames (Orlov, 2014a; 2014b; 2014c).

If some experiment will confirm the existence of kinematical maximal acceleration $\alpha$, then it will have certainly crucial consequences for Einstein theory of gravity because this theory does not involve this factor. Also the cosmological theories constructed on the basis of the original 
Einstein equations will require modifications. The so called Hubble constant will be changed and the scenario of the accelerating universe modified.

Also the standard model of particle physics and supersymmetry theory will require generalization because they does not involve the maximal acceleration constant. It is not excluded that also the theory of parity nonconservation will be modified by the maximal acceleration constant. In such a way the particle laboratories have perspective applications involving the physics with maximal acceleration. Many new results can be obtained from the old relativistic results having the form of the mathematical objects involving function $f(v / c)$. The derived formulas with uniform acceleration can be applied and verified in case of the uniform equivalent gravity according to the principle of equivalence.

The prestige problem in the modern theoretical physics - the theory of the Unruh effect, or, the existence of thermal radiation detected by accelerated observer - is in the development (Fedotov et al., 2002) and the serious statement, or comment to the relation of this effect to the maximal acceleration must be elaborated. The analogical statement is valid for the Hawking effect in the theory of black holes. Maximal acceleration, determines the maximal black hole mass where the mass of the black hole is restricted by maximal acceleration of a body falling in the gravity field of the black hole.

It is not excluded that the maximal acceleration constant $\alpha$ will be confirmed by the DIRAC experiment in CERN, or by International Linear Collider (ILC).

Let us remark in conclusion that it is possible to extend and modify quantum field theory by maximal acceleration. It is not excluded that the kinematic maximal acceleration constant will enable to reformulate the theory of renormalization.

\section{REFERENCES}

Caianiello, E. R. (1981). Is There maximal acceleration? Lett. Nuovo Cimento, 32, 65 ; ibid. (1992). Revista del Nuovo Cimento, 15, No. 4.

Eisenhart, L. P. Continuous groups of transformations, (Princeton, 1943).

Fedotov, A. M., Narozhny, N. B. , Mur, V. D. and Belinski, V. A. (2002). An example of a uniformly accelerated particle detector with non-Unruh response, arXiv: hep-th/0208061.

Lambiase, G., Papini, G. and Scarpetta, G. (1998). Maximal acceleration limits on the mass of the Higgs boson, arXiv:hep-ph/9808460; ibid. (1999). Nuovo Cimento B, 114, 189-197.

Lightman, A. P., Press W. H., Price, R. H. and Teukolsky S. A. Problem book in relativity and gravitation, (Princeton University Press, 1975 ).

Orlov, E. P. (2014a). Transformation of space-time during relativistic rectilinear acceleration motion in the framework of additional measurement, FIAN, Lebedev Physical Institute, preprint No. 3.

Orlov, E. P. (2014b). Comparison analysis of transformation of space-time during relativistic rectilinear acceleration motion, FIAN, Lebedev Physical Institute, preprint No. 7.

Orlov, E. P. (2014c). Transformation of space-time between systems of coordinates connected with opposite charges accelerated by electric field, FIAN, Lebedev Physical Institute, preprint No. 19.

Papini, G. (2003). Revisiting Caianiello's maximal acceleration, e-print quant-ph/0301142.

Pardy, M. (1969; 1974). The group of transformations for accelerated systems, Jan Evangelista Purkyně University, Brno (unpublished).

Pardy, M. (2003). The space-time transformation and the maximal acceleration, arXiv:grqc/ 
0302007.

Pardy, M. (2004). The space-time transformation and the maximal acceleration, Spacetime \& Substance Journal, 1(21), (2004), pp. 17-22.

Pardy, M. (2005). Creativity leading to discoveries in particle physics and relativity, arXiv:physics/0509184

Selleri, F. (1997). The relativity principle and nature of time, Foundations of Physics, 27, No. $11,1527$.

Schuetz, Ch. P. (2003). The DIRAC experiment at CERN, arXiv:hep-ph/0305121v1 12 May 2003 , 